\title{
Assessing Explanatory Models for Common Mental Disorders
}

\author{
Kamaldeep Bhui, M.D.; Katja Rüdell, Ph.D.; \\ and Stefan Priebe, Ph.D.
}

Objectives: This article addresses the clinical implications of 3 questions: (1) Can a simple checklist, suitable for use by practitioners, assess all components of explanatory models (EMs) for mental distress? (2) Are perceived causes of mental distress actually related to treatment preferences? (3) Are EMs influenced uniquely by ethnic group, or are they more closely associated with the presence of common mental disorders?

Method: From February 2003 to January 2004, we investigated EMs for mental distress among 79 Bangladeshi, 85 black Caribbean, and 97 white British people who reported difficulties in the preceding month. EMs were assessed by a self-report checklist that inquired about the identity, causes, timeline, consequences, controllability, and preferred treatments for mental distress. Common mental disorders were assessed using the Clinical Interview Schedule (revised).

Results: Independent of ethnic group, people with common mental disorders were more likely to give spiritual causal explanations (odds ratio $[\mathrm{OR}]=3.1,95 \% \mathrm{CI}=1.9$ to 4.9 ), to report behavioral $(\mathrm{OR}=2.2,95 \% \mathrm{CI}=1.3$ to 3.8$)$ and financial consequences $(\mathrm{OR}=3.3,95 \% \mathrm{CI}=1.8$ to 6), and to prefer complementary treatments $(\mathrm{OR}=4.6,95 \% \mathrm{CI}=2.3$ to 9.1$)$. Compared with black Caribbean and white British subjects, Bangladeshi subjects more often gave spiritual or physical causal explanations. Compared with white British subjects, Bangladeshi and black Caribbean subjects preferred medical and spiritual treatments, whereas white British subjects, as compared with the other 2 ethnic groups, preferred self-management and social treatments. Causal explanations did not always relate to the corresponding treatment preferences.

Conclusions: EMs can be assessed by a simple checklist, show variations by ethnic group, and are associated with common mental disorders. Identifying EMs may strengthen the assessment of common mental disorders.

(J Clin Psychiatry 2006;67:964-971)
Received Jan. 9, 2005; accepted Dec. 6, 2005. From the Centre for Psychiatry, Barts and the London School of Medicine, Queen Mary University of London.

The study was funded by the Joint Research Board of

St. Bartholomew's and The Royal London Charitable Trust.

The authors report no additional financial or other relationship relevant to the subject of this article.

We are grateful to general practitioners and the subjects who were all very generous with their thoughts and time.

Corresponding author and reprints: Kamaldeep Bhui, M.D., Centre for Psychiatry, Wolfson Institute of Preventive Medicine, Barts and The London School of Medicine, Old Anatomy Building, Charterhouse Square, London EC1M 6BQ (e-mail:K.s.bhui@qmul.ac.uk).

$\mathbf{P}$ ersonal explanations for mental distress, also called explanatory models (EMs), may explain variations in the assessment and management of mental disorders. ${ }^{1,2}$ Research studies show that differences between patients' EMs and the medical professions' explanations for conditions such as asthma, diabetes, leprosy, tuberculosis, anxiety, and depression explain ethnic variations in assessment and recognition of these medical conditions. ${ }^{3-10}$ Yet, clinicians may not appreciate the significance of EMs for mental disorders when these draw on social, religious, or supernatural attributions. ${ }^{7-10}$ For example, an Ethiopian woman's complaints of having a "snake in her leg" may be misunderstood to be delusional, instead of appropriately understood as an idiomatic expression of having problems with her mother-in-law. ${ }^{10}$ Patients' perceived causes of common mental disorders are strong predictors of functional impairment and short-term prognosis and are better predictors than diagnoses or symptoms. ${ }^{11}$ EMs for depression that include an external locus are also indicators of a poor prognosis. ${ }^{12}$

Assessing EMs is now recommended in routine practice in order to improve the cultural competency of assessment, diagnostic validity, and therapeutic relationships. ${ }^{1,13,14}$ It is important to reconcile patients' and the doctors' divergent EMs about diagnosis, treatment, and prognosis; this is a particular challenge in culturally diverse societies. ${ }^{13,14}$ Assessing EMs ensures a better insight into the patient's personal experience of illness. Jaspers ${ }^{15}$ argued this was essential in order to assess the significance of psychopathology, by ensuring a better understanding of the patients' subjective experience of illness in its social context. However, previous research has focused mainly on causal explanations rather than all com- 
Bhui et al.

\begin{tabular}{|c|c|c|}
\hline Domain & Items (numbers and examples) & Thematic Groups Used in Analyses \\
\hline Identity & $\begin{array}{l}41 \text { labels for the identity of their condition: } \\
\text { examples include visual deficiency, crying, screaming, } \\
\text { bodily weakness, violence, being mute, and hallucinations }\end{array}$ & Physical health, mental health, behavioral changes \\
\hline Causes & $\begin{array}{l}40 \text { possible causes of their distress: for example, bereavement, } \\
\text { test of faith, substance use, family problems, fate, the climate, } \\
\text { genetic, financial, poison, and viruses }\end{array}$ & $\begin{array}{l}\text { Social, spiritual, behavioral, climate related, } \\
\text { medical/physical health }\end{array}$ \\
\hline Timeline & Cyclical or duration of condition & Cyclical, more than or less than 6 months \\
\hline Consequences & $\begin{array}{l}26 \text { items on the consequences of distress: for example, feeling sad, } \\
\text { being locked up, losing their job, fearing going out, pain, } \\
\text { and using alcohol }\end{array}$ & $\begin{array}{l}\text { Psychological, behavioral, financial, social, } \\
\text { physical health }\end{array}$ \\
\hline Preferred interventions & $\begin{array}{l}\text { 18-item checklist that includes talking to your friends, } \\
\text { talking to your general practitioner, dance, taking medication, } \\
\text { prayer, exercise, and use of alcohol }\end{array}$ & $\begin{array}{l}\text { Self-care, social care, complementary, spiritual } \\
\text { treatment such as prayer, medical/physical health }\end{array}$ \\
\hline
\end{tabular}

ponents of EMs, and only a few studies included several ethnic groups and applied the same methods of measuring mental disorders and EMs for valid comparisons.

In this article, we consider how assessing EMs may strengthen the clinical assessment of common mental disorders. We investigated EMs in 3 distinct ethnic groups living in an inner city urban area, using the same study methods. Three questions were addressed: (1) Can a simple checklist, suitable for use by practitioners, assess all components of EMs for mental distress? (2) Are causal explanations related to treatment preferences? (3) Are EMs influenced uniquely by ethnic group, or are they also influenced by the presence of a common mental disorder?

\section{METHOD}

\section{Assessment of Explanatory Models}

The term explanatory model arose from anthropological critiques of psychiatric practice. We use the term explanatory model in this article rather than similar terms such as illness model or illness representation that sometimes feature in medical, psychological, and sociological research. All of these terms and related theoretical models draw on personal explanations for illness and share 5 building blocks., ${ }^{2,16}$ These include the patient's own understanding of what to call their illness (illness identity) based on the dominant complaints, the perceived cause of the illness, the timeline or course of the condition, the consequences of the condition and perceived controllability, and perceived effective treatments.

From February 2003 to January 2004, we assessed EMs using the Barts Explanatory Model Inventory (BEMI), which was developed empirically from published accounts of expressions of distress from the world literature. ${ }^{17}$ These published accounts were reviewed and subjected to qualitative analyses in order to cluster the data into key thematic categories. ${ }^{17,18}$ An independent second researcher clustered items into named themes and found good agreement $(\kappa=0.8)$. This process relied on qualitative conceptually driven approaches to validation, rather than only relying on statistical tests. ${ }^{18,19}$

Clinicians do not usually have the time and resources to undertake a detailed and unstructured exploration of EMs or qualitative data analysis. Therefore, we modified the interview version to produce a more useful short self-report checklist for use in clinical settings and survey research (BEMI-C ${ }^{20}$; see Table 1). Endorsement of items was higher for the checklist version compared with the interview version, which documented only spontaneous EMs. ${ }^{17}$ The BEMI-C showed good face and content validity, test-retest reliability (Pearson correlation coefficients between 0.78 and 0.95$)$, and interrater agreement of clustered items $(\kappa=0.8)$. Subjects were asked to read and endorse all items that describe their perceptions about their condition (see Table 1, column 1). These items correspond to a smaller number of thematic categories (column 3 in Table 1) that were used to summarize the key findings and for statistical analyses.

\section{Assessment of Common Mental Disorders}

We assessed mental health status on the Clinical Interview Schedule (revised version). ${ }^{21}$ This is a validated measure of common mental disorders (anxiety and depression) assessed on the basis of 14 symptoms in a structured interview format; it has been used in cross-cultural settings and in national and international research. Scores of 12 or more indicate clinically significant morbidity consistent with an ICD or DSM diagnosis. ${ }^{1,21,22}$ This is the threshold we used in the analyses. Continuous measures of common mental disorders are recognized to reflect true population distributions. ${ }^{21-23}$ Although such measures are not widely used because of poor clinical utility, ${ }^{23}$ ascertainment of significant mental disorders on the Clinical Interview Schedule shows good agreement with clinical diagnoses and the need for clinical intervention. ${ }^{21}$ 


\section{Sociodemographic and Health Information}

We also asked about age, gender, employment (manual/nonmanual), receipt of any benefits, attendance of higher education (college, university after the age of 16 years), accommodation (owned, rented private, council tenant), and the self-reported presence of a chronic physical health problem. The full interview was available in English, Bengali, and Sylheti and was administered to those who reported "a problem or difficulty that distressed them in the last month."

\section{Sampling}

Ethnicity was defined by self-ascription in accord with 2001 national census categories, on the basis of ethnic group recorded in the primary care records, and by verification at interview. This classification is used in national and local surveys and in research and policy development in the United Kingdom. Although there are variations in social class, education, and migration experiences within these groups, these groups are seen as cogent ethnic groups for research and reflect immigration patterns to the United Kingdom. ${ }^{24,25}$

Community surveys report that over $98 \%$ of all ethnic groups are registered in primary care. ${ }^{26}$ Subjects were randomly sampled patients from primary care registers (4 practices) wherever their ethnic group was specified as black Caribbean, Bangladeshi, or white British (response rates: $29 \%, 44 \%$, and $46 \%$, respectively). Ethnicity information was not always recorded in primary care records. Therefore, to recruit more representative samples from a wide range of ages and demographic strata and across genders, we also recruited attendees of non-health agencies in the community. ${ }^{27}$ Local non-health-related community venues (15 sites) included colleges, community groups, and social venues. Ethnic origin was confirmed at interview. The study received ethical approval from the local ethical review board (East London and City Health Authority Ethics Committee). There were no enticements to participate.

We recruited 125 white British people, 116 black Caribbean people, and 122 Bangladeshi people. Of these subjects, 97 white British subjects, 85 black Caribbean, and 79 Bangladeshi subjects endorsed our screening question (Have you had any problem or difficulty in the preceding month?) and so proceeded to complete the BEMI checklist. The screening question was broad in order to include all who may have suffered from some emotional distress so that we could then ask about their explanatory models. We also recorded duration of residence in the United Kingdom. Subjects proceeding to interview showed no significant differences from those not interviewed by gender, ethnic group, recruitment from general practitioner or community venue, duration of stay in the United Kingdom, occupation, or accommodation status.

\section{Statistical Analyses}

Demographic characteristics were tabulated by ethnic group (Table 2). EMs were tabulated by ethnic group and common mental disorder (Table 3). We undertook $\chi^{2}$ and Kruskal-Wallis tests of significance for these data.

Previous studies emphasized causal explanations as determinants of treatment choice. We assessed this by calculating pairwise correlations between causal explanations and preferred treatments. Correlations between causal explanations with preferred treatments were reported only if they reached a significance of $p<.01$, to make allowance for the number of significance tests for ethnic group.

We also undertook logistic regression analyses to assess whether EMs were associated with ethnic group or with common mental disorders (a binary variable). ${ }^{21}$ We built 5 separate models corresponding to each of the domains of EMs: identity, causal explanations, timeline, consequences, and treatment. Logistic regression models took account of the cluster-sampled data, using the "cluster" command in Stata 5.0 (StataCorp LP, College Station, Tex.). Although not all potential confounders were associated with common mental disorders, we adjusted for them all in order to ensure the most parsimonious findings. We applied the conservative Bonferroni correction and report findings reaching a significance of $\mathrm{p}<.003$ for any one model. Where components of EMs were associated with the common mental disorder in regression, we calculated sensitivities, specificities, and positive and negative predictive validities for them as individual components of EMs and as a group in order to evaluate their future value in the assessment and recognition of common mental disorder (Table 4$){ }^{28}$

\section{RESULTS}

\section{Sample Characteristics}

Black Caribbean subjects were more likely to be recruited from the community, were more often women, were less likely to own their houses, and were the least likely to have a common mental disorder compared with the other ethnic groups (see Table 2). Bangladeshi subjects were least likely to receive financial welfare support, to be in paid employment, or to have received higher education; Bangladeshis were most likely to be born outside of the United Kingdom and have a common mental disorder (Table 2).

\section{Ethnic Variations of Explanatory Models}

There were significant ethnic differences related to EMs (Table 3; percentages in the Total columns):

Identity of the distress. Black Caribbean people were less likely to label their distress as a physical illness compared with white British and Bangladeshi people $\left(\chi^{2}=21\right.$, $\mathrm{df}=2, \mathrm{p}<.001)$. White British people were more likely to 


\begin{tabular}{|c|c|c|c|c|c|c|c|c|c|}
\hline \multirow[b]{2}{*}{ Explanatory Models } & \multicolumn{3}{|c|}{ White British } & \multicolumn{3}{|c|}{ Bangladeshi } & \multicolumn{3}{|c|}{ Black Caribbean } \\
\hline & $\begin{array}{c}\text { Noncases } \\
(\mathrm{N}=61), \\
\mathrm{N}(\%)\end{array}$ & $\begin{array}{c}\text { Cases } \\
(\mathrm{N}=36) \\
\mathrm{N}(\%)\end{array}$ & $\begin{array}{c}\text { Totals } \\
(\mathrm{N}=97), \\
\mathrm{N}(\%)\end{array}$ & $\begin{array}{c}\text { Noncases } \\
(\mathrm{N}=22), \\
\mathrm{N}(\%)\end{array}$ & $\begin{array}{c}\text { Cases } \\
(\mathrm{N}=57) \\
\mathrm{N}(\%) \\
\end{array}$ & $\begin{array}{c}\text { Total } \\
(\mathrm{N}=79) \\
\mathrm{N}(\%)\end{array}$ & $\begin{array}{c}\begin{array}{c}\text { Noncases } \\
(\mathrm{N}=63), \\
\mathrm{N}(\%)\end{array}\end{array}$ & $\begin{array}{c}\text { Cases } \\
(\mathrm{N}=20) \\
\mathrm{N}(\%)\end{array}$ & $\begin{array}{c}\text { Total }^{\mathrm{a}} \\
(\mathrm{N}=83), \\
\mathrm{N}(\%) \\
\end{array}$ \\
\hline \multicolumn{10}{|l|}{ Identity } \\
\hline Reports physical health problems & $53(86.9)$ & $36(100.0)$ & $89(91.8)$ & $18(81.8)$ & $57(100.0) \dagger$ & 75 (94.9) & $40(63.5)$ & $20(100.0) \dagger$ & $60(72.3) \ddagger$ \\
\hline Reports mental health problems & $47(77.0)$ & $35(97.2) \dagger$ & $82(84.5)$ & $16(72.7)$ & $57(100.0) *$ & $73(92.4)$ & $48(76.2)$ & $20(100.0)$ & $69(81.9)$ \\
\hline Reports behavioral problems & $41(67.2)$ & $36(100.0) \div$ & $77(79.4)$ & $10(45.5)$ & $45(78.9)^{*}$ & $55(69.6)$ & $31(49.2)$ & $20(100.0) \ddagger$ & $51(61.5)^{*}$ \\
\hline \multicolumn{10}{|l|}{ Causal Explanation } \\
\hline Psychosocial & $54(88.5)$ & $36(100.0)$ & $90(92.8)$ & $21(95.5)$ & $47(82.5)$ & $68(86.1)$ & $47(74.6)$ & $19(95.0)$ & $66(78.6) *$ \\
\hline Spiritual & $6(9.8)$ & $10(27.8)^{*}$ & $16(16.5)$ & $11(50.0)$ & $48(84.2) \dagger$ & $58(74.7)$ & $12(19.1)$ & $13(65.0) \div$ & $26(31.0) \div$ \\
\hline Behavioral & $17(27.9)$ & $19(52.8)^{*}$ & $36(37.1)$ & $3(13.6)$ & $10(17.5)$ & $13(16.5)$ & $11(17.5)$ & $11(55.0) \dagger$ & $22(26.5) \dagger$ \\
\hline Weather & $5(8.2)$ & $7(19.4)$ & $12(12.4)$ & $3(13.6)$ & $9(15.8)$ & $12(15.2)$ & $3(4.8)$ & $10(50.0) \ddagger$ & $13(15.7)$ \\
\hline Physical & $11(18.0)$ & $13(36.1)^{*}$ & $24(24.7)$ & $6(27.3)$ & $34(59.7)^{*}$ & $40(50.6)$ & $7(11.1)$ & $11(55.0) \ddagger$ & $18(21.7)+$ \\
\hline Situational/environmental & $13(21.3)$ & $20(55.6) \dagger$ & $33(31.4)$ & $5(22.7)$ & $18(31.6)$ & $23(29.1)$ & $14(22.2)$ & $13(65.0) \dagger$ & $27(32.1)$ \\
\hline \multicolumn{10}{|l|}{ Timeline } \\
\hline Cyclical/coming and going & $50(82.0)$ & $30(83.3)$ & $80(82.5)$ & $8(40.0)$ & $30(53.6)^{\mathrm{b}}$ & $38(50.0)$ & $36(57.1)$ & $13(65.0)$ & $49(59.0) \dagger$ \\
\hline Lasted more than 6 months & $23(37.7)$ & $27(75.0) \div$ & $50(51.6)$ & $12(54.5)$ & $49(86.0) \dagger$ & $61(77.2)$ & $39(60.9)$ & $13(65.0)$ & $52(62.7) \dagger$ \\
\hline \multicolumn{10}{|l|}{ Consequences } \\
\hline Psychological & $47(77.1)$ & $36(100.0) \dagger$ & $83(85.6)$ & $19(86.4)$ & $57(100.0) \dagger$ & $76(96.2)$ & $42(66.7)$ & $20(100.0) \dagger$ & $62(74.7) \div$ \\
\hline Social & $16(26.2)$ & $23(63.9) \div$ & $39(40.2)$ & $7(31.8)$ & $32(56.1)$ & $39(49.4)$ & $24(38.1)$ & $13(65.0)^{*}$ & $37(44.6)$ \\
\hline Physical & $19(31.1)$ & $18(50.0)$ & $37(38.1)$ & $6(27.3)$ & $34(59.7) \dagger$ & $40(50.6)$ & $16(25.4)$ & $14(70.0) \div$ & $30(36.1)$ \\
\hline Behavioral & $22(36.1)$ & $21(58.3)^{*}$ & $43(44.3)$ & $5(22.7)$ & $25(43.9)$ & $30(38.0)$ & $9(14.3)$ & $12(60.0) \ddagger$ & $21(25.3)$ \\
\hline Financial & $9(14.8)$ & $13(36.1)^{*}$ & $22(22.7)$ & $4(18.2)$ & $25(43.9)^{*}$ & $29(36.7)$ & $10(15.9)$ & $11(55.0) \div$ & $21(25.3)^{*}$ \\
\hline \multicolumn{10}{|l|}{ Treatments, Control, and Cure } \\
\hline Self-management helpful & $47(77.1)$ & $32(88.9)$ & $79(81.4)$ & $12(54.5)$ & $35(61.4)$ & $47(59.5)$ & $26(41.3)$ & $16(80.0) \dagger$ & $42(50.6) *$ \\
\hline Social treatment helpful & $51(83.6)$ & $28(77.8)$ & $79(81.4)$ & $11(50.0)$ & $33(57.9)$ & $44(55.7)$ & $23(36.5)$ & $14(70.0) \dagger$ & $38(45.2) \div$ \\
\hline Medical treatment helpful & $13(21.3)$ & $10(27.8)$ & $23(23.7)$ & $5(22.7)$ & $28(49.1)^{*}$ & $33(41.8)$ & $16(25.4)$ & $9(45.0)$ & $26(30.1)$ \\
\hline Alternative treatment helpful & $7(11.5)$ & $11(30.6)^{*}$ & $18(18.6)$ & $0(0)$ & $7(12.3)$ & $7(8.9)$ & $3(4.8)$ & $8(40.0) \div$ & $11(13.3)$ \\
\hline Spiritual treatment helpful & $6(9.8)$ & $2(5.6)$ & $8(8.3)$ & $6(27.3)$ & $18(31.6)$ & $24(30.1)$ & $20(31.8)$ & $10(50.0)$ & $30(35.7) \div$ \\
\hline
\end{tabular}

\begin{tabular}{lccccccc}
\hline \multicolumn{7}{l}{ Table 4. Associations of Explanatory Models and Common Mental Disorder in Logistic Regression Models } \\
\hline Explanatory Model & Odds Ratio & $95 \%$ CI & p Value & Sensitivity & Specificity & PPV & NPV \\
\hline Spiritual causes & 3.1 & 1.9 to 4.9 & $<.001$ & 62.9 & 80.2 & 71.0 & 73.6 \\
Behavioral consequences & 2.2 & 1.3 to 3.8 & .003 & 51.3 & 75.3 & 61.7 & 66.8 \\
Financial consequences & 3.3 & 1.8 to 6.0 & .001 & 43.3 & 84.3 & 68.1 & 65.8 \\
Complementary treatments & 4.6 & 2.3 to 9.1 & $<.001$ & 23.0 & 93.2 & 72.2 & 61.0 \\
Composite: positive to any of the above & $\ldots$ & $\ldots$ & $\ldots$ & 92.0 & 56.2 & 61.9 & 90.1 \\
\hline
\end{tabular}

Abbreviations: NPV $=$ negative predictive validity, $\mathrm{PPV}=$ positive predictive validity.

$\mathrm{p}<.001)$ more helpful in controlling distress, whereas significantly smaller proportion of white British individuals held this view.

\section{Causal Explanations and Preferred Treatments}

Medical causal explanations were significantly correlated with preferring medical treatments, but only among black Caribbean (pairwise correlation: 0.44) and white British subjects (pairwise correlation: 0.35). In contrast to the other ethnic groups, black Caribbean subjects with medical causal explanations were also more likely to seek nonmedical treatments (pairwise correlation for social treatments: 0.25 ; for self-directed treatment: 0.31 ; and for complementary treatments:
0.30). Among white British subjects, spiritual causal explanations were also related to a preference for medical treatment (pairwise correlation: 0.28). Among black Caribbean subjects, spiritual causal explanations were correlated with self-management (pairwise correlation: 0.3 ) and a preference for complementary treatments (pairwise correlation: 0.35 ).

\section{Common Mental Disorders and Explanatory Models}

Some components of EMs were more prevalent among people with common mental disorders when compared with those without common mental disorders (Table 3); these differences varied by ethnic group. Only significant findings from regression models are shown in Table 4. 
Independent of ethnic group, people with common mental disorders were more likely to give spiritual causal explanations (Table 4), to report significant behavioral and financial consequences of their illness, and to prefer complementary treatments. Spiritual causal explanations had high sensitivity and specificity for common mental disorders (Table 4). A composite assessment that takes account of "spiritual" explanations, behavioral and financial consequences, and the use of complementary treatments had a higher sensitivity but a lower specificity than any single component of the EM (Table 4).

\section{DISCUSSION}

\section{Use of Checklist}

The BEMI-C, a checklist used in this study to assess all components of EMs, yielded plausible results. The findings varied-in some aspects-between ethnic groups and showed substantial associations with the presence of common mental disorders. The checklist is brief and simple to use. It should be possible to administer the checklist not only in large scale research, but also in routine care where longer interviews are not feasible.

\section{Ethnic Patterns of EMs and the Link Between Causal Explanation and Treatment Preference}

We confirmed ethnic variations of the perceived nature, cause, consequences, and preferred treatments for common mental disorders. Causal explanations were not always predictive of preferred interventions in any one ethnic group. Pluralistic help seeking, the popularity of self-management and complementary treatments, and psychosocial causal explanations were common, irrespective of ethnic group.

We found that white British people more often held social causal explanations and preferred self-management. Among those with physical causal explanations, black Caribbean and white British people sought out medical treatment, but Bangladeshi people did not. Our findings of more spiritual or supernatural explanations offered by black Caribbean and Bangladeshi subjects with common mental disorders are consistent with previous findings for patients with schizophrenia in the same ethnic groups ${ }^{8}$ and patients presenting to primary care with common mental disorders. ${ }^{6,29}$

Although psychological causal explanations and mental disorder as the identity of distress were common across all ethnic groups, we found that Bangladeshis more often proposed psychological consequences of their distress. This contradicts previous findings that South Asians less often report psychological distress and that they more often present somatic symptoms. ${ }^{29-32}$ Our study does show that psychosocial explanations are common and often coexistent with other causal explanations. This coexpression of different EMs and the propensity to not report psychosocial explanations in the presence of somatic complaints may partly explain the diagnostic difficulties of recognizing mental disorders across ethnic groups. ${ }^{1,29-32}$

\section{Improving Psychiatric Assessments}

A better understanding of a patient's EM will help clinicians manage divergent patient and professional treatment expectations. ${ }^{8}$ Resolving differences without conflict is one of the characteristics of traditional and complementary practitioners and is said to explain the popularity of such practices. Therefore, tackling divergent EMs earlier rather than after a period of nonadherence or dropout from treatment can be expected to enhance the therapeutic alliance. Doing so avoids frustration and may be experienced by the patient as a more useful encounter in which their beliefs have been heard and understood, rather than dismissed. This in turn may engender more trust and offers a behavioral model for the patient that shows a willingness to work with an alternative EM.

Asking about patients' EMs can enhance the quality of clinical assessment, especially across cultural boundaries where there is scope for both underdiagnosis and overdiagnosis. The cultural consultation model recommends the reconciliation of divergent patient and professional EMs without conflict. ${ }^{14,29}$ Addressing these when treatment is commenced may improve treatment adherence through improvement of the patients' understanding of their condition and the clinicians' understanding of the need for psychoeducational approaches. ${ }^{14}$

Although EMs vary between ethnic groups, they also vary within each ethnic group, and so clinical assessment should be of individual patients' EMs to avoid overreliance on stereotyped group differences. The BEMI-C offers a reliable method for doing this. While the identification of a medical diagnosis is to be based on symptom criteria as defined in DSM and ICD, information on patients' EMs may be a useful component of a comprehensive assessment and may be used to establish a positive therapeutic alliance with patients from diverse backgrounds. This probably applies to all ethnic groups.

\section{Spiritual Explanations and \\ Complementary Treatments}

Spiritual causal explanations, the expectation of serious consequences, and seeking complementary treatments all suggest the presence of a common mental disorder. In our study, spiritual explanations referred to religious, astrological, magical, and ancestor influences as well as destiny, bad luck, and punishment from God. The high correlation of spiritual explanations with common mental disorders may reflect that spiritual explanations emerge following nonresponse to conventional treatments or that those with spiritual explanations for common mental disorders do not seek out help from con- 
ventional sources of help. Complementary treatments are based on a theory of body and mind that is more congruent with a spiritual explanation of distress. Therefore, such treatments may be associated with common mental disorders if patients are themselves identifying interventions that are in accord with their own EMs.

\section{Strengths and Limitations}

Our study is situated in an inner city area where ethnic groups can comprise up to $45 \%$ of the local population. Although we did not examine the effects of ethnic density on EMs, in high ethnic density areas people may hold on to traditional attitudes and EMs may be more resistant to change. We did take account of first and second generation effects by adjusting for place of birth in analyses. However, other than place of birth and educational level, we had no direct measures of acculturation and patterns of EMs by integrated, assimilated, or traditional cultural identities.

Although EMs of mental disorder can be incorporated into diagnostic assessments as a way of developing new culturally valid instruments, ${ }^{11,32,33}$ only a few of these studies tested associations between EMs and mental disorders. ${ }^{11,33}$ We reported that mental distress was associated with financial and behavioral consequences, spiritual causal explanations, and a preference for complementary treatments. Where these complaints are all present, patients are likely to meet criteria for an anxiety or depressive disorder. Previous work shows that assessment of patients' perceived causes of common mental disorders may improve psychiatric assessment. ${ }^{11}$ However, we included aspects of EMs that most studies do not explore, for example, reported consequences, controllability, and treatment options.

This was a cross-sectional case-control study that showed clinically relevant associations. It is possible that the components of EMs that were associated with common mental disorder emerged as a consequence of having a common mental disorder, or their presence marks a more vulnerable group who go on to develop common mental disorder. It is known that EMs can change over time, and we did not follow up subjects, so this study cannot unravel the causal sequence. We did not investigate mechanisms that favor the expressions of specific EMs. For example, in countries where mental health problems are stigmatized, somatic EMs may be more common. ${ }^{11}$

\section{Directions for Future Clinical Research}

The processes that generate inequalities are in part context driven and related to social inequalities. However, particular cultural idioms and metaphors in clinical consultations coupled with this social disadvantage may collectively contribute to inequalities of access to services as well as differing experiences and outcomes from services. The empirical data on social disadvantage and treatment experiences of ethnic groups show similar patterns in the United States and the United Kingdom, albeit for different ethnic groups. ${ }^{34,35}$ Future research should include prospective designs and other ethnic groups. Future research might explore how EMs influence psychiatric assessments in live consultations and assess other outcomes such as social functioning and quality of life. Contextual variables such as ethnic density, neighborhood cohesion, and acculturation may also be important to explore. Larger samples will allow stratification by gender, age, and social variables, by racial and ethnic groups, and by cultural subgroups. The modification of illness perceptions is an effective intervention to improve adherence to treatments and clinical outcomes in chronic illnesses. ${ }^{35-37}$ Thus, specific trials of interventions targeting EMs of mental distress may help improve adherence and future engagement in different clinical samples in mental health care.

\section{REFERENCES}

1. Jacob KS, Bhugra D, Lloyd KR, et al. Common mental disorders, explanatory models and consultation behaviour among Indian women living in the UK. J R Soc Med 1998;91:66-71

2. Kleinman A. Patients and Healers in the Context of Culture. Berkeley and Los Angeles, Calif: University of California Press; 1980

3. Griffiths C, Foster G, Barnes N, et al. Specialist nurse intervention to reduce unscheduled asthma care in a deprived multiethnic area: the east London randomised controlled trial for high risk asthma (ELECTRA). BMJ 2004;328:144. Epub 2004 Jan 12

4. Griffiths C, Kaur G, Gantley M, et al. Influences on hospital admission for asthma in south Asian and white adults: qualitative interview study. BMJ 2001;323:962-966

5. Glasman D, Finlay WM, Brock D. Becoming a self therapist: using cognitive behavioral therapy for recurrent depression and/or dysthymia after completing therapy. Psychol Psychother 2004;77:335-351

6. Lloyd KR, Jacob KS, Patel V, et al. The development of the Short Explanatory Model Interview (SEMI) and its use among primary-care attenders with common mental disorders. Psychol Med 1998;28: 1231-1237

7. Weiss M. Explanatory Model Interview Catalogue (EMIC): framework for comparative study of illness. Transcultural Psychiatry 1997;24: 235-263

8. McCabe R, Priebe S. Explanatory models of illness in schizophrenia: comparison of four ethnic groups. Br J Psychiatry 2004;185:25-30

9. Eisenbruch M. Classification of natural and supernatural causes of mental distress: development of a Mental Distress Explanatory Model Questionnaire. J Nerv Ment Dis 1990;178:712-719

10. Schreiber S. Migration, traumatic bereavement and transcultural aspects of psychological healing: loss and grief of a refugee woman from Begameder County in Ethiopia. Br J Med Psychol 2001;68:135-142

11. Henningsen P, Jokobsen T, Schiltenwolf M, et al. Somatization revisited: diagnosis and perceived causes of common mental disorders. J Nerv Ment Dis 2005; 193:85-92

12. Bann CM, Parker CB, Bradwejn J, et al. Assessing patient beliefs in a clinical trial of hypericum perforatum in major depression. Depress Anxiety 2004,20:114-122

13. Tseng WS. Clinician's Guide to Cultural Psychiatry. London, UK: Academic Press; 2003:219-289

14. Kirmayer LJ, Groleau D, Guzder J, et al. The cultural consultation model: a model of mental health services for multicultural societies. Can J Psychiatry 2003;48:145-153

15. Jaspers C. General Psychopathology. Hoenig J, Hamilton MW, trans. Baltimore, Md: Johns Hopkins University Press; 1997

16. Leventhal $\mathrm{H}$. Fear appeals and persuasion: the differentiation of a motivational construct. Am J Public Health 1971;61:1208-1224 
Bhui et al.

17. Rudell K. The development of a cross cultural framework and new instrument to assess cultural variations in perceptions of distress: Barts Explanatory Model Inventory (BEMI). [PhD thesis]. London, UK: University of London; 2005

18. Pope C, Ziebland S, Mays N. Qualitative research in health care: analysing qualitative data. BMJ 2000;320:114-116

19. Bhui K, Bhugra D. Explanatory models for mental distress: implications for clinical practice and research. Br J Psychiatry 2002;181:6-7

20. Protocol for Barts Explanatory Model Interview. Available at: http:// www.kent.ac.uk/psychology/department/people/abstracts/rudellk/ bemi04.pdf. Access verified May 12, 2006

21. Lewis G, Pelosi AJ, Araya R, et al. Measuring psychiatric disorder in the community: a standardized assessment for use by lay interviewers. Psychol Med 1992;22:465-486

22. Jacob KS, Everitt BS, Patel V, et al. The comparison of latent variable models of non-psychotic psychiatric morbidity in four culturally diverse populations. Psychol Med 1998;28:145-152

23. Kendell R, Jalbensky A. Distinguishing between validity and utility of psychiatric diagnoses. Am J Psychiatry 2003;160:4-12

24. Singleton N, Bumpstead R, O'Brien M, et al. Psychiatric morbidity among adults living in private households. UK Office for National Statistics; 2000. Available at: http://www.statistics.gov.uk/downloads/ theme health/psychmorb.pdf. Access verified April 14, 2006

25. Bhui K, Stansfeld S, McKenzie K, et al. Racial/ethnic discrimination and common mental disorders among workers: findings from the EMPIRIC Study of Ethnic Minority Groups in the United Kingdom. Am J Public Health 2005;95:496-501

26. Johnson P. Black and Minority Ethnic Groups in England: The Second Health and Lifestyles Survey. London, UK: Health Education Authority; 2000

27. Spring M, Westermeyer J, Halcon L, et al. Sampling in difficult to access refugee and immigrant communities. J Nerv Ment
Dis 2003;191:813-819

28. Swets JA, Pickett RM. Evaluations of Diagnostic Systems: Methods of Signal Detection Theory. New York, NY: Academic Press; 1982

29. Bhui K, Bhugra D, Goldberg D. Causal explanations of distress and general practitioners' assessments of common mental disorder among Punjab and English attendees. Soc Psychiatry Psychiatr Epidemiol 2002;37:38-45

30. Nambi SK, Prasad J, Singh D, et al. Explanatory models and common mental disorders among patients with unexplained somatic symptoms attending a primary care facility in Tamil Nadu. Natl Med J India 2002; 15:331-335

31. Gillam SJ, Jarman B, White P, et al. Ethnic differences in consultation rates in urban general practice. BMJ 1989;299:953-957

32. Bhui K, Bhugra D, Goldberg D, et al. Cultural influences on the prevalence of common mental disorder, general practitioners' assessments and help-seeking among Punjabi and English people visiting their general practitioner. Psychol Med 2001;31:815-825

33. Patel V. Explanatory models of mental illness in sub-Saharan Africa. Soc Sci Med 1995;40:1291-1298

34. US Department of Health and Human Services. Mental Health: Culture, Race, and Ethnicity: A Supplement to Mental Health: A Report of the Surgeon General. Rockville, Md: US Dept Health Human Services, Substance Abuse and Mental Health Services Administration, Center for Mental Health Services; 2001

35. Bhui K, McKenzie K, Gill P. Delivering mental health services for a diverse society. BMJ 2004;329:363-364

36. Petrie KJ, Cameron LD, Ellis CJ, et al. Changing illness perceptions after myocardial infarction: an early intervention randomized controlled trial. Psychosom Med 2002;64:580-586

37. Horne R, Graupner L, Frost S, et al. Medicine in a multi-cultural society: the effect of cultural background on beliefs about medications. Soc Sci Med 2004;59:1307-1313 